Research Paper

\title{
YTH domain family: potential prognostic targets and immune- associated biomarkers in hepatocellular carcinoma
}

\author{
Miaomiao Liu', Zijin Zhao², Yuan Cai ${ }^{3}$, Peng Bi ${ }^{3}$, Qiuju Liang ${ }^{4}$, Yuanliang Yan ${ }^{4,5}$, Zhijie Xu ${ }^{3}$ \\ ${ }^{1}$ Department of Nuclear Medicine (PET-CT Central), Xiangya Hospital, Central South University, Changsha, China \\ ${ }^{2}$ Department of Neurosurgery, Xiangya Hospital, Central South University, Changsha, China \\ ${ }^{3}$ Department of Pathology, Xiangya Hospital, Central South University, Changsha, China \\ ${ }^{4}$ Department of Pharmacy, Xiangya Hospital, Central South University, Changsha, China \\ ${ }^{5}$ National Clinical Research Center for Geriatric Disorders, Xiangya Hospital, Central South University, Changsha, \\ China
}

Correspondence to: Zhijie Xu; email: xzj1322007@csu.edu.cn

Keywords: hepatocellular carcinoma, YTH domain family, expression profiles, infiltrating immune cells, methylation

Received: July 19, 2021

Accepted: October 26, 2021

Published: November 8, 2021

Copyright: (c) 2021 Liu et al. This is an open access article distributed under the terms of the Creative Commons Attribution License (CC BY 3.0), which permits unrestricted use, distribution, and reproduction in any medium, provided the original author and source are credited.

\begin{abstract}
Hepatocellular carcinoma (HCC) is the most common high malignancy with insidious onset, invasive fastgrowing, high recurrence rate and fatality. YTH domain family plays essential roles in development of HCC. However, the biological function of YTH domain family in HCC have not been clarified. Here, through evaluating the expression profiles of YTH domain family, we found that upregulated YTHDF1 might be more significant and valuable in development and progression of HCC. There was a strong correlation between YTHDC1, YTHDF1 and YTHDF2 and pathological stage of HCC patients. Kaplan-Meier plotter revealed that HCC patients with high level of YTHDF1 and YTHDF2 were highly related to a shorter overall survival time, and low level of YTHDF1 ( $p=$ 0.0017) has an important association with a longer progression-free survival time. Genetic alterations using cBioPortal revealed that the alteration rates of YTHDF3 were the highest. We also found that the functions of YTH domain family were linked to several cancer-associated pathways, including peptidyl-serine modification, peptidyl-tyrosine modification and negative regulation of cellular component movement. TIMER database indicated that the YTH domain family had a strong relationship with the infiltration of six types of immune cells (macrophages, neutrophils, CD8+ T-cells, B-cells, CD4+ T-cells and dendritic cells). Next, Ualcan databases revealed that the global methylation levels of YTHDC1 was higher in HCC patients, while YTHDF2 was lower in HCC patients. In conclusion, our findings will enhance the understanding of YTH domain family in HCC pathology, and provide novel insights into YTH-targeted therapy for HCC patients.
\end{abstract}

\section{INTRODUCTION}

Hepatocellular carcinoma (HCC) has become one of the most common and aggressive malignant disease to bring about worldwide concern, which ranks as the sixth of morbidity and the third of mortality in all malignant tumors $[1,2]$. The onset of this malignant disease is usually indetectable and insidious resulting in having developed into middle and advanced stage [3]. The therapeutic outcome of HCC is unfavorable leading to the 5 years survival rate of HCC is less than $19 \%$ [4]. Consequently, exploration of novel molecular markers will play an essential role for improving the prognosis of HCC patients.

Accumulating statistics indicate that among a series of RNA modification adenosines, N6-methyladenosine (m6A) plays the most significant part in modulating numerous cellular processes including cancer biological functions [5, 6]. Meanwhile, a recent study revealed that 
m6A is linked to the pathogenesis and development of HCC by regulating several targeted genes [7]. The YT521-B homology (YTH) domain family members, including YTHDC1, YTHDC2, YTHDF1, YTHDF2 and YTHDF3, are the main "m6A readers" [8]. Furthermore, YTH domain proteins are mostly enrolled in the tumorigenesis and tumor progression $[9,10]$. However, the specific mechanisms of YTH domain family members in HCC development and disease progression still require further confirmation and research.

Despite the YTH domain family plays significant role in cancer development, the systematic analysis of YTH domain family expression profiles and functions in HCC were still insufficient. In our study, we devoted to explore the expression features and potential biological functions of YTH domain family in HCC patients (Supplementary Table 1).

\section{RESULTS}

\section{Aberrant expression of YTH domain family in HCC patients}

We used TIMER database to detect the expression levels of YTH domain family members. And the result showed that all of YTH domain family members were significantly raised in various malignant tumors (Supplementary Figure 1). Then, the mRNA expression levels of YTH domain family members in HCC tissues and normal tissues were evaluated by the Wanderer database. We found that the expression level of YTHDC1 $(\mathrm{p}=1.83 \mathrm{E}-04)$ was significantly downregulated in HCC patients. While, the expression level of YTHDF1 ( $\mathrm{p}=$ 1.55E-03) was higher in $\mathrm{HCC}$ tissues than in normal tissues (Figure 1A). Then, the GEPIA database was used to evaluate the gene expression of YTH domain family members in HCC tissues and normal tissues again. The analysis showed that expression levels of YTHDF1 ( $\mathrm{p}<$ $0.05)$, YTHDF2 ( $<$ < 0.05) and YTHDF3 ( $<$ < 0.05) were significantly upregulated in HCC patients (Figure 1B). Further, the expression of YTH domain family members in HCC tissues and adjacent tissues were analyzed with HCCDB database. We found that the expression of YTHDC2 $(\mathrm{p}=4.93 \mathrm{E}-04)$, YTHDF1 $(\mathrm{p}=1.76 \mathrm{E}-26)$, YTHDF2 ( $\mathrm{p}=3.49 \mathrm{E}-07)$, and YTHDF3 ( $\mathrm{p}=3.21 \mathrm{E}-30)$ in HCC tissues were higher than in adjacent tissues (Table 1). Therefore, based on the above evaluation in these different databases, upregulated YTHDF1 might be more significant and valuable in development and progression of HCC.

Then, we explored the association between the expression profiles of YTH domain family and the
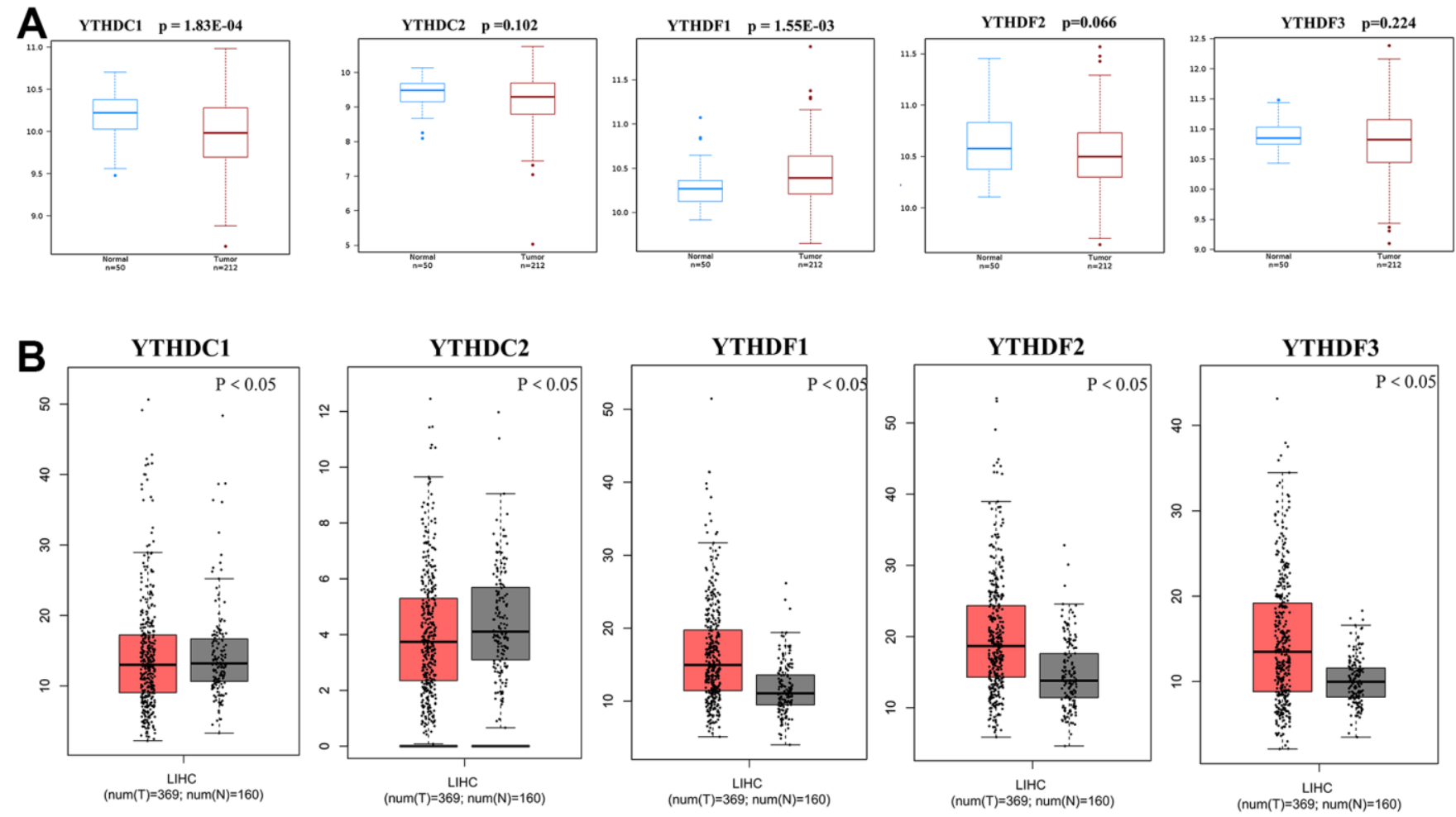

Figure 1. Differential mRNA expression analysis of the YTH domain family in HCC and normal tissues. (A) The expression profiles were collected from the Wanderer databases. (B) The expression profiles were collected from the GEPIA databases. 
Table 1. The expression of YTH domain family members in HCC tissues and adjacent tissues from the HCCDB database.

\begin{tabular}{lccccccc}
\hline Dataset & Gene & Type & Nums & Mean & STD & P-value & IQR \\
\hline \multirow{4}{*}{ YTHDC1 } & HCC & 212 & 3.103 & 0.3624 & $3.23 \mathrm{E}-01$ & 0.4225 \\
& & Adjacent & 177 & 3.069 & 0.3108 & & 0.38 \\
& YTHDC2 & HCC & 212 & 2.864 & 0.479 & $4.93 \mathrm{E}-04$ & 0.5525 \\
& & Adjacent & 177 & 2.713 & 0.3645 & & 0.46 \\
\multirow{3}{*}{ HCCDB18 } & YTHDF1 & HCC & 212 & 3.884 & 0.3942 & $1.76 \mathrm{E}-26$ & 0.52 \\
& & Adjacent & 177 & 3.447 & 0.3555 & & 0.45 \\
& \multirow{2}{*}{ YTHDF2 } & HCC & 212 & 4.175 & 0.4425 & $3.49 \mathrm{E}-07$ & 0.585 \\
& & Adjacent & 177 & 3.972 & 0.3301 & & 0.42 \\
& \multirow{2}{*}{ YTHDF3 } & HCC & 212 & 4.049 & 0.5341 & $3.21 \mathrm{E}-30$ & 0.7025 \\
& & Adjacent & 177 & 3.505 & 0.3027 & & 0.31 \\
\hline
\end{tabular}

pathological stages of HCC patients. As shown in Figure 2, YTHDC1 expression $(\mathrm{p}=0.0144)$, YTHDF1 expression $(\mathrm{p}=0.000138)$ and YTHDF2 expression $(\mathrm{p}$ $=0.0206)$ displayed a positive correlation with pathological stage. These data suggested that aberrant expression of YTH domain family members might participate in disease progression in HCC patients.

\section{The prognostic value of the YTH domain family in HCC patients}

Then, The Kaplan-Meier plotter was used to evaluate the prognostic value of YTH domain family for patients with HCC. The data of overall survival (OS) curves are shown in Figure $3 \mathrm{~A}$. We found that a high transcriptional level of YTHDF1 $(\mathrm{p}=0.0013)$ and YTHDF2 $(\mathrm{p}=0.0068)$ were highly related to a shorter OS time. At the same time, we evaluated the prognostic significance of YTH domain family on the progression-free survival (PFS) of HCC patients. From Figure 3B, we know that low level of YTHDF1 $(\mathrm{p}=0.0017)$ has an important association with a longer PFS time. Thus, the YTHDF1 might be more important and influential in prognostic value to $\mathrm{HCC}$ patients.

\section{Genetic alteration and functional enrichment analyses of YTH domain family in HCC patients}

To further analyze the alteration profiles of YTH domain members in patients with $\mathrm{HCC}$, we conducted a series of surveys as follows. First, we used the cBioPortal database to evaluate the genetic alterations of YTH domain family. As shown in Figure 4A, we confirmed that the alternation rate of YTHDF3 was the highest in 24\% of cases, whereas the lowest was for YTHDC1, which was only 6\%. In addition, YTHDC2, YTHDF1 and YTHDF2 mutations occurred in $7 \%, 18 \%$ and $10 \%$ of the samples, respectively (Figure 4A).

Next, Using HCCDB database to evaluate the associated molecules of YTH domain family, we found that the interactive molecules of all members had altered significantly between tumor tissues and adjacent normal tissues, respectively (Figure 4B). Additionally, we used cBioPortal to extract 203 most frequently altered genes that were significantly linked to the YTH domain family in HCC patients (Supplementary Table 2). The data indicated that several hub genes, such as tumor protein p53 (TP53), mammalian target of rapamycin (MTOR) and mitogen-activated protein kinase 1 (MAPK1), were
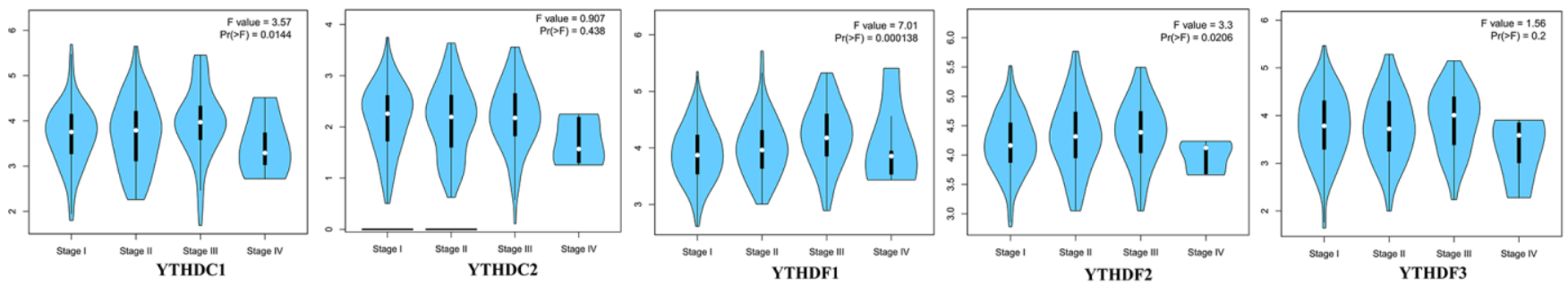

Figure 2. The relationship between the expression of the YTH domain family and the pathological stage of HCC patients (GEPIA). GEPIA databases were used to evaluate the correlations of the YTH domain family with the pathological stage of HCC patients. 

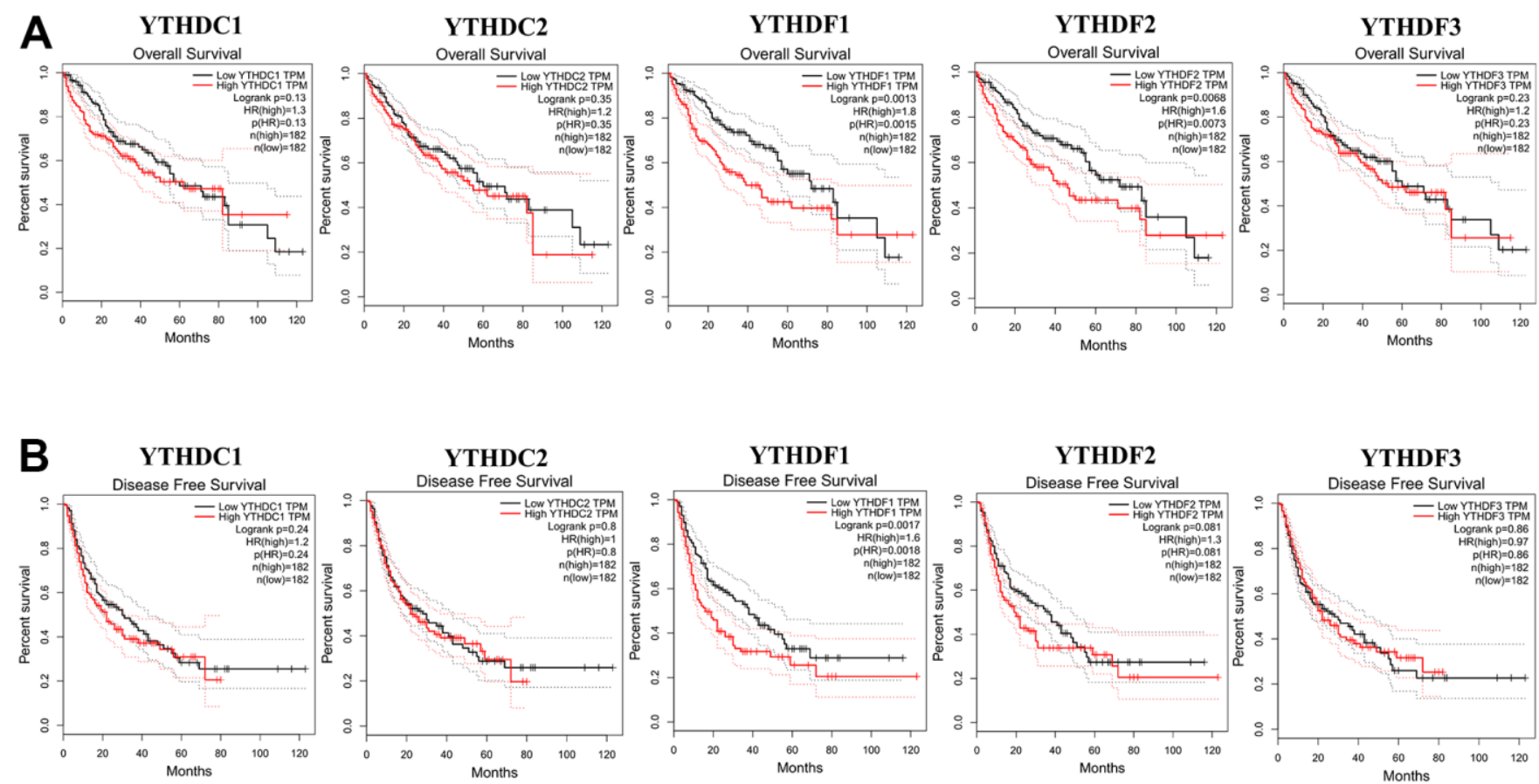

Figure 3. The correlations of YTH domain family expression with OS and PFS in HCC patients. Kaplan-Meier plotter was used to assess the correlation of YTH domain family members with the patients' OS (A) and PFS time (B).

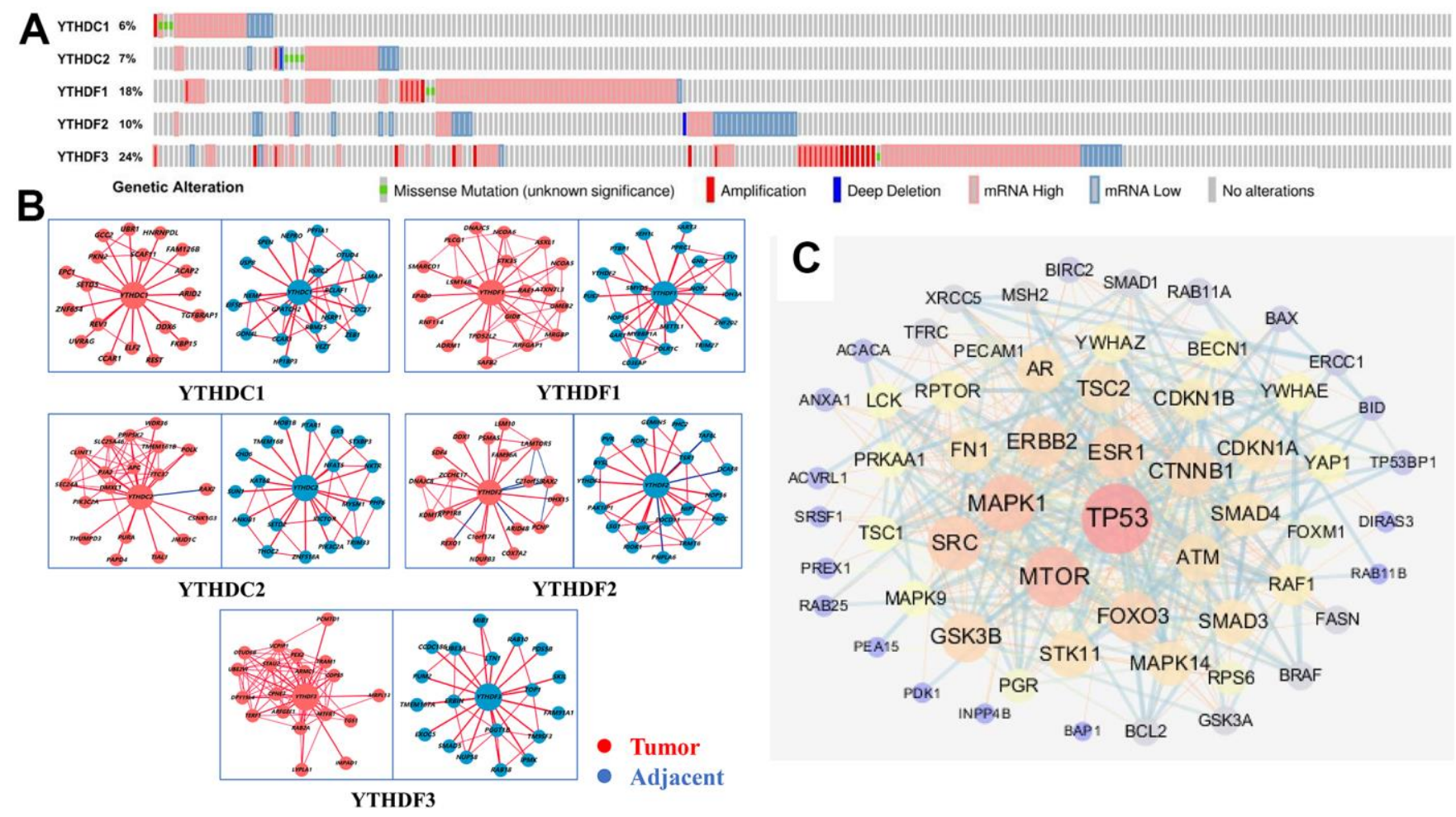

Figure 4. Genetic alterations and interaction analyses of the YTH domain family in HCC patients. (A) Genetic alteration of the YTH domain family in HCC patients analyzed with cBioPortal. (B) The HCCDB database was used to analyze the YTH domain family coexpressed genes in HCC and adjacent tissue samples. (C) The most frequently altered genes identified from cBioPortal that are linked to the YTH domain family in HCC patients. 
closely linked to the biological processes in YTH domain family in HCC patients (Figure 4C). Above data suggested that YTH domain family participated in the regulation of various signaling pathways involved in HCC biology.

In our study, we applied the WebGestalt database to evaluate the potential biological functions of YTH domain family in the occurrence and development of HCC. As shown in the Gene Ontology (GO) term analysis (Figure 5A), we know that the most highly enriched biological process (BP) category was response to stimulus, followed by metabolic process, biological regulation, etc. In the cellular component (CC) categories, membrane, nucleus, cytosol, membraneenclosed lumen, protein-containing complex, endomembrane system and vesicle were highly accumulated. In the molecular function (MF) categories, the YTH domain family members were mainly enriched in protein binding, nucleotide binding, nucleic acid binding, ion binding and transferase activity. Meanwhile, the Kyoto Encyclopedia of Genes and Genomes (KEGG) pathway analysis results are shown in Figure 5B. We can conclude that several cancer-associated pathways, including peptidyl-tyrosine modification, peptidyl-serine modification and negative regulation of cellular component movement, were strongly linked to the potential biological functions of YTH domain family in the occurrence and development of HCC.

\section{The roles of YTH domain family on immune cell infiltration}

TIMER database was used to explore the relationship between immune cell infiltration and expression profiles of YTH domain family (Figure 6). The expression of YTHDC1 was positively linked to the infiltration of $\mathrm{B}$ cells $($ Cor $=0.313, \mathrm{p}=3.10 \mathrm{e}-09)$, CD8 $+\mathrm{T}$ cells $(\mathrm{Cor}=$ $0.296, \mathrm{p}=2.30 \mathrm{e}-08), \mathrm{CD} 4+\mathrm{T}$ cells $(\mathrm{Cor}=0.412, \mathrm{p}=$ $1.61 \mathrm{e}-15)$, macrophages $(\mathrm{Cor}=0.435, \mathrm{p}=3.37 \mathrm{e}-17$ ), neutrophils $(\mathrm{Cor}=0.518, \mathrm{p}=4.58 \mathrm{e}-25)$ and dendritic cells (Cor $=0.448, \mathrm{p}=3.22 \mathrm{e}-18$ ) (Figure 6A). There was a positive correlation between YTHDC2 expression and the infiltration of B cells (Cor $=0.202, p=1.59 \mathrm{e}-04)$, CD8+ $\mathrm{T}$ cells $(\mathrm{Cor}=0.159, \mathrm{p}=3.26 \mathrm{e}-03), \mathrm{CD} 4+\mathrm{T}$ cells $(\mathrm{Cor}=$ $0.300, \mathrm{p}=1.40 \mathrm{e}-08)$, macrophages $(\mathrm{Cor}=0.343, \mathrm{p}=$ 7.18e-11), neutrophils $($ Cor $=0.449, \mathrm{p}=1.71 \mathrm{e}-18)$ and dendritic cells (Cor $=0.268, \mathrm{p}=5.11 \mathrm{e}-07$ ) (Figure 6B). YTHDF1 expression positively related with the infiltration of purity (Cor $=0.126, \mathrm{p}=1.80 \mathrm{e}-02)$, B cells $(\mathrm{Cor}=0.349, \mathrm{p}=2.70 \mathrm{e}-11), \mathrm{CD} 8+\mathrm{T}$ cells $(\mathrm{Cor}=0.239, \mathrm{p}$ $=8.22 \mathrm{e}-06), \mathrm{CD} 4+\mathrm{T}$ cells $(\mathrm{Cor}=0.418, \mathrm{p}=5.11 \mathrm{e}-16)$, macrophages $($ Cor $=0.480, p=5.23 \mathrm{e}-21)$, neutrophils (Cor $=0.447, \mathrm{p}=2.25 \mathrm{e}-18)$ and dendritic cells (Cor $=0.401, \mathrm{p}=1.36 \mathrm{e}-14)$ (Figure 6C). We concluded that
YTHDF2 expression was positively correlated with B cells $($ Cor $=0.191, \mathrm{p}=3.70 \mathrm{e}-04), \mathrm{CD} 8+\mathrm{T}$ cells $(\mathrm{Cor}=$ 0.201, $\mathrm{p}=1.83 \mathrm{e}-04), \mathrm{CD} 4+\mathrm{T}$ cells $($ Cor $=0.373, \mathrm{p}=$ 8.97e-13), macrophages (Cor $=0.418, \mathrm{p}=7.23 \mathrm{e}-16$ ), neutrophils $(\mathrm{Cor}=0.480, \mathrm{p}=3.02 \mathrm{e}-21)$ and dendritic cells (Cor $=0.344, \mathrm{p}=6.71 \mathrm{e}-11$ ) (Figure 6D). We also found that the expression of YTHDF3 was positively related to the infiltration of B cells (Cor $=0.143, p=8.03 \mathrm{e}-03$ ), CD4+ T cells $($ Cor $=0.303, p=9.44 \mathrm{e}-09)$, macrophages (Cor $=0.209, \mathrm{p}=9.71 \mathrm{e}-05)$, neutrophils (Cor $=0.334, \mathrm{p}=$ 1.87e-10) and dendritic cells (Cor $=0.204, p=1.57 \mathrm{e}-04$ ) (Figure 6E). In addition, the Cox proportional hazard model revealed that $B$ cells $(p=0.021), C D 8+T$ cells $(p=$ $0.017)$, dendritic cells $(\mathrm{p}=0.005)$ and YTHDF1 $(\mathrm{p}=$ 0.005) had a strong relationship with HCC patient prognosis (Table 2).

\section{Methylation level of the YTH domain family}

Furthermore, we analyzed the methylation levels of YTH domain family by searching Ualcan database. We found that the methylation levels of YTHDC1 ( $p<$ 1E-12) was higher in HCC patients than in normal cases; while YTHDF2 ( $\mathrm{p}=1.478 \mathrm{E}-06)$ was lower in HCC patients (Figure 7). These downregulated methylation values might explain their difference in expression levels to some extent.

\section{DISCUSSION}

YTH domain family members were the m6A "reader" proteins which were firstly identified. M6A modification can be recognized by YTH domain family proteins in a methylation-dependent manner, which is identified by sequence comparison and is found in 174 different proteins expressed in eukaryotes [11]. There are five YTH domain-containing proteins are found in human genome, which can be classified into three categories: YTHDC1, YTHDC2 and YTHDF1-3 [12]. YTH domain family proteins are collected by m6A and linked to several mRNA metabolism including mRNA splicing, translation, nuclear export, and mRNA degradation [13, 14]. Therefore, YTH domain family proteins may be involved in many tumor physiological processes $[8,15]$. In literature, YTHDF1, an oncogene in $\mathrm{HCC}$, is reported to promote the epithelial mesenchymal transition (EMT) of HCC cells by accelerating the translation of snail family transcriptional repressor 1 (SNAI1) [16]. Meanwhile, Overexpression of YTHDF1 is also presented to promote HCC cell proliferation, differentiation and metastasis via accelerating the transcription of frizzled5 mRNA through an m6A-dependent manner [17]. Functioning as a tumor suppressor, high level of YTHDF2 could inhibit the proliferation and neoplasia by degrading EGFR mRNA of HCC cells in an m6A- 
dependent manner [18]. However, YTHDF2 also plays a repressive role in $\mathrm{HCC}$ by promoting the reduction of inflammatory cytokines interleukin (IL)-11 [19]. However, the detailed functions and mechanisms of YTH domain family in HCC have not been fully explored and explained.

In our study, according to the TIMER, Wanderer, GEPIA and HCCDB database, we concluded that the expression levels of YTH domain family are different between HCC and normal tissues. Especially, YTHDF1 might be more significant and valuable to be as an oncogene in development and progression of HCC. Additionally, we also performed the relationship between expression levels of YTH domain family and HCC pathological stage. We found that the expression level of YTHDC1, YTHDF1 and YTHDF2 were increased with the HCC stage progressed, suggesting that these three proteins might participate in disease progression in $\mathrm{HCC}$ patients. Moreover, HCC patients with high expression of YTHDF1 and YTHDF2 had a poor OS time. HCC patients with high expression of YTHDF1 had a poor PFS value. These databases implied that YTH domain family is crucial for the progression and survival of $\mathrm{HCC}$ patients. However, more specific studies about the expression and prognostic values of YTH domain family in patients with HCC needs to be further explored.

To better understand the potential mechanism of YTH domain family in HCC, we performed GO and KEGG pathway enrichment analysis to explore the function of YTH domain family. We found that the biological
A Bar chart of Biological Process categories

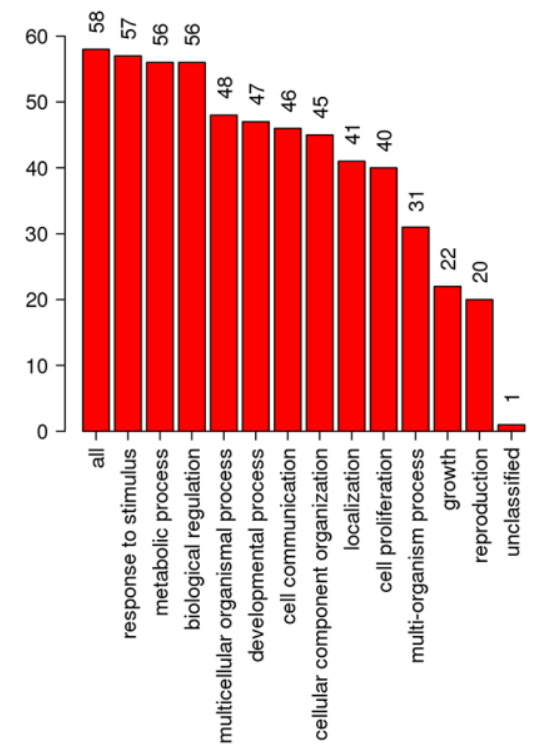

Bar chart of Cellular Component categories

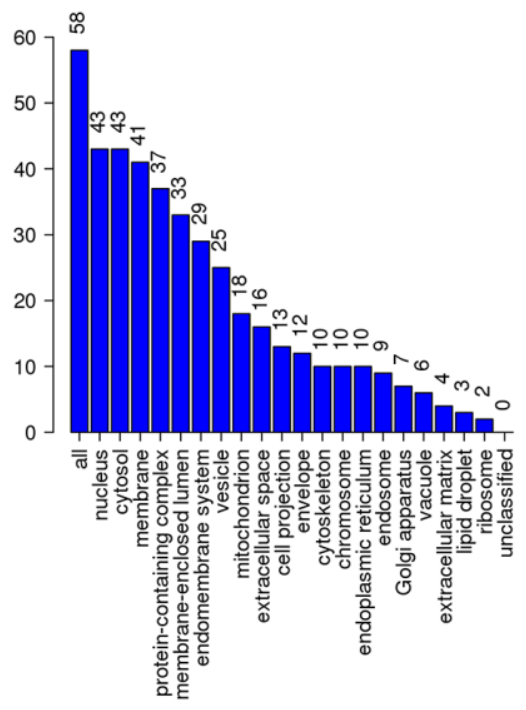

Bar chart of Molecular Function categories

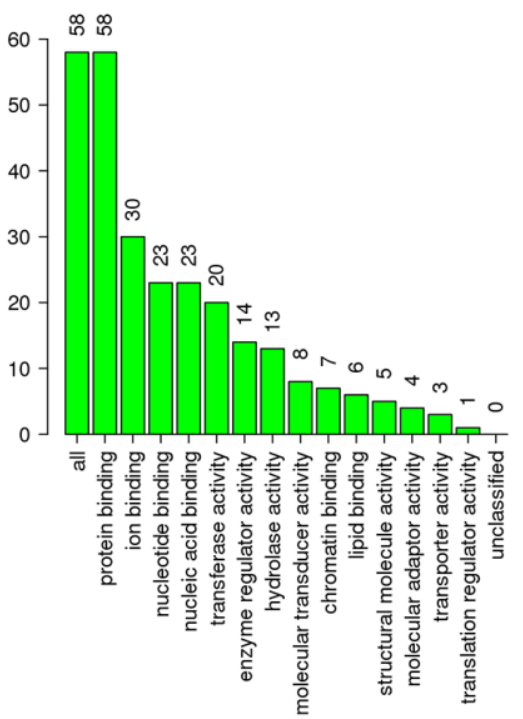

B

FDR $\leq 0.05=$ FDR $>0.05$

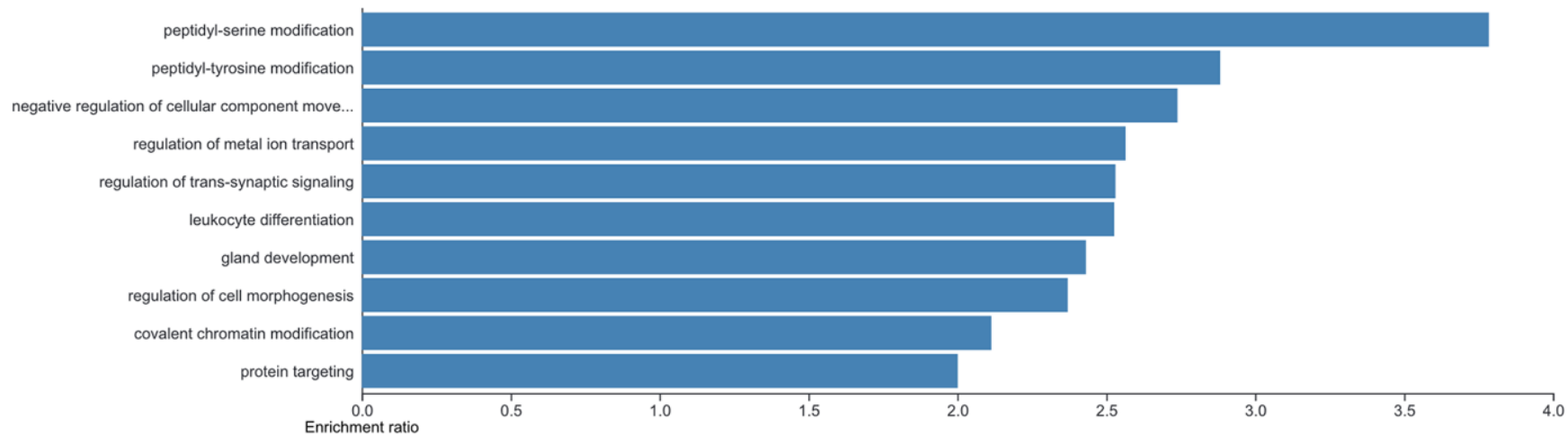

Figure 5. The biological pathways of the YTH domain family were evaluated by the WebGestalt database. (A) Bar plot of GO enrichment in cellular components, biological processes, and molecular functions. (B) The bar plot of KEGG enrichment. 
functions of YTH domain family participated primarily in the posttranslational modification pathways, such as peptidyl-serine modification and peptidyl-tyrosine modification. A previous study has shown that serine biosynthesis pathway and tyrosine relative kinase inhibitors play significant roles in the regulation of cancer progress and immunotherapy for HCC [20, 21]. In addition, the KEGG enrichment pathway analysis revealed that YTH domain family could also participate in several cancer-associated pathways, such as negative regulation of cellular component movement pathway [8,
17-19]. These findings suggest that YTH domain family could be drawn into the progression of HCC through regulating protein posttranslational modification pathways.

Immune molecules play a critical role in the regulating tumor responses [22-24]. A growing number of researches have focused on the interactions between tumors and immune cells $[25,26]$ and found that the expression levels of YTH domain family were strongly related to the immune cells infiltration, including

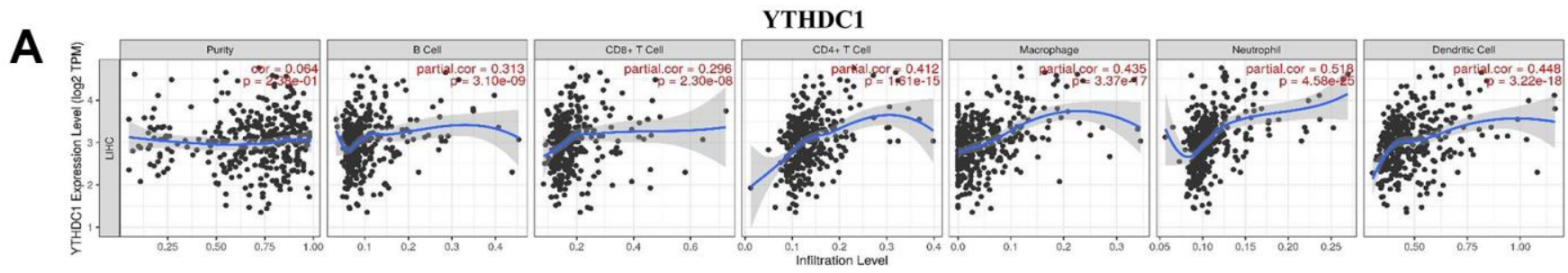

YTHDC2

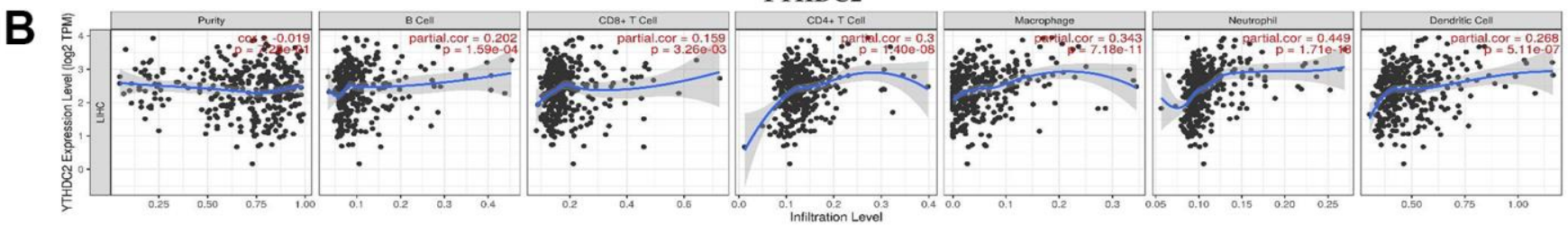

YTHDF1
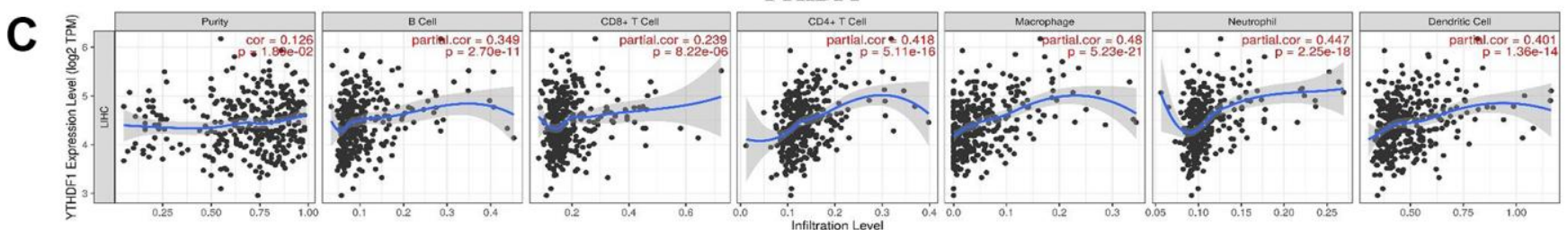

YTHDF2
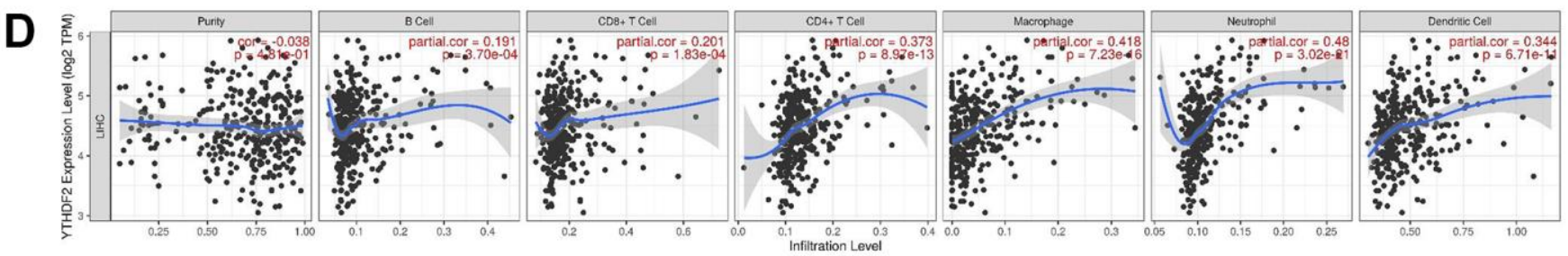

YTHDF3
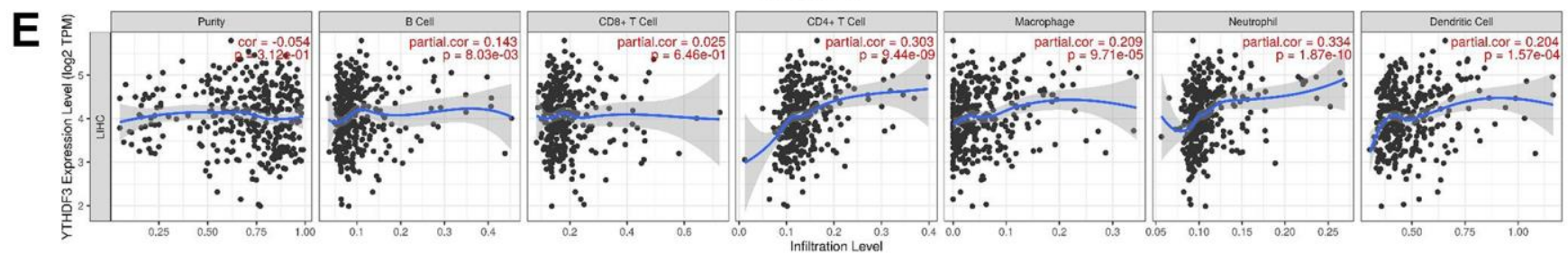

Figure 6. The relationship between immune cell infiltration and the expression of the YTH domain family. (A-E) The Timer database was used to analyze the effect of (A) YTHDC1, (B) YTHDC2, (C) YTHDF1, (D) YTHDF2, (E) YTHDF3 on the abundance of immune cells in HCC patients. 
Table 2. The cox proportional hazard model of TYH domain family and six types of immune cells in HCC patients from timer database.

\begin{tabular}{|c|c|c|c|c|c|c|}
\hline & Coef & HR & 95\%CI_l & 95\%CI_u & P.value & Sig \\
\hline B_cell & -8.087 & 0.000 & 0.000 & 0.288 & 0.021 & * \\
\hline CD8_Tcell & -5.768 & 0.003 & 0.000 & 0.359 & 0.017 & * \\
\hline CD4_Tcell & -4.773 & 0.008 & 0.000 & 4.642 & 0.138 & \\
\hline Macrophage & 3.593 & 36.356 & 0.228 & 5799.076 & 0.165 & \\
\hline Neutrophil & -0.241 & 0.786 & 0.000 & 75634.618 & 0.967 & \\
\hline Dendritic & 5.056 & 157.029 & 4.755 & 5185.355 & 0.005 & $* *$ \\
\hline YTHDC1 & -0.482 & 0.618 & 0.356 & 1.071 & 0.086 & \\
\hline YTHDC2 & -0.217 & 0.805 & 0.542 & 1.194 & 0.281 & \\
\hline YTHDF1 & 0.763 & 2.145 & 1.258 & 3.659 & 0.005 & $* *$ \\
\hline YTHDF2 & 0.518 & 1.678 & 1.001 & 2.815 & 0.050 & \\
\hline YTHDF3 & 0.021 & 1.022 & 0.715 & 1.460 & 0.907 & \\
\hline
\end{tabular}

B cells, CD4+ T cells, macrophages, neutrophils and dendritic cells. Our result aligned with other researches, indicated that immune cells infiltration could have important effects on the immunotherapy and clinical outcomes of cancers [27, 28]. A recent study has pointed that the m6A regulators were significantly associated with tumor immune microenvironment in HCC [29]. For example, YTHDF2 expression was correlated with some infiltrating immune cells in HCC, such as B cells, CD8+ T cells, $\mathrm{CD} 4+\mathrm{T}$ cells, macrophages, neutrophils, and DCs
[30]. In addition, Shi et al. [31] found that YTHDF1 facilitated NSCLC cell proliferation and xenograft tumorigenesis by regulating the translational efficiency of several immune checkpoints. In our study, we found that the expression profiles of YTHDF1 is significantly linked to the infiltration of B cells, CD8+ T cells, dendritic cells. These data indicated that the YTH domain family could regulate the infiltration of immune cells, which may trigger an intense immune response of infiltrating immune cells in the tumor environment.

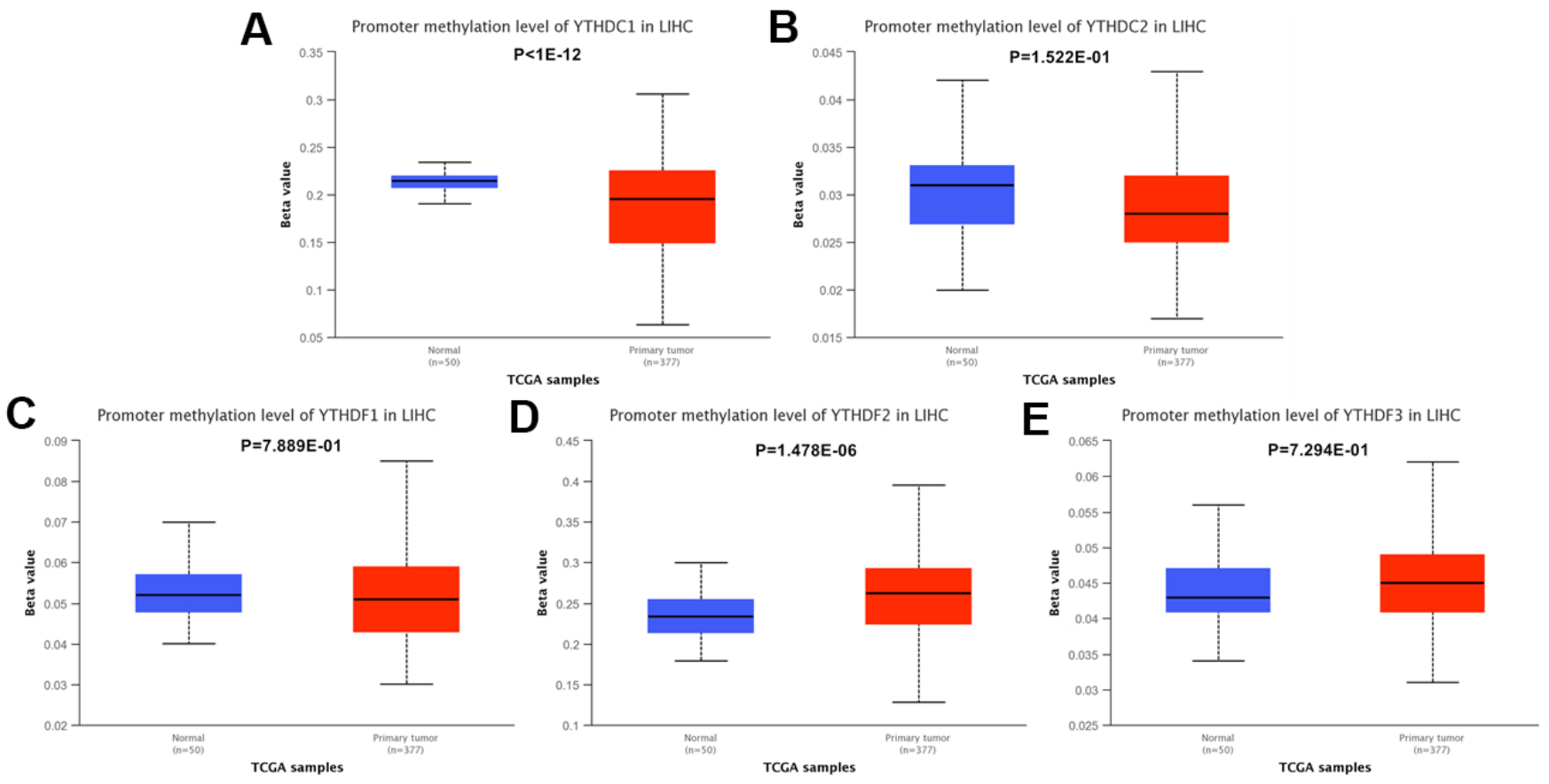

Figure 7. The methylation levels of the YTH domain family in HCC tissues. (A-E) The methylation values of (A) YTHDC1, (B) YTHDC2, (C) YTHDF1, (D) YTHDF2, (E) YTHDF3 were evaluated using the Ualcan database. 
In the present study, there still exist some limitations. Firstly, the statistics we analyzed were mainly from the databases, therefore, we should present more in vitro and in vivo experiments to identify the functions and mechanisms of YTH domain family in HCC. Secondly, the study did not conduct some survey concerning the therapeutic outcome of HCC patients. Thus, the outcomes need to be validated and amended in the future multicenter, large-scale clinical trials. Finally, it is important to explore prognostic effects of the YTH domain family on HCC patients in order to improve their application values in clinic.

In conclusion, we comprehensively analyzed the profiles of YTH domain family in HCC from the perspective of bioinformatics, including expression levels, prognostic values and immune response. The results showed the YTH domain family, especially YTHDF1, possessed great potentiality as therapeutic targets and prognostic biomarkers in HCC via regulating multiple mechanisms. These findings might help clinicians design effective therapeutic strategies to improve the treatment effect and prognosis of $\mathrm{HCC}$ patients.

\section{MATERIALS AND METHODS}

\section{Wanderer}

Wanderer, an intuitive Web tool, which permits realtime access and visualization of gene expression and DNA methylation profiles from TCGA using gene targeted queries [32]. Wanderer was applied to evaluate the YTH domain family expression profiles in tumor tissues and normal tissues.

\section{GEPIA}

Gene Expression Profiling Interactive Analysis (GEPIA) is a database that is designed to help end users fully understand gene expression from a more holistic perspective [33]. We used "Single Gene Analysis" in GEPIA to evaluate the expression levels of YTH domain family members in tumor tissues compared with normal tissues. We also used this database to evaluate the effects of YTH domain family in pathological stage, prognosis and so on. The p-value was $<0.05$.

\section{HCCDB}

HCCDB is a one-stop online database to explore HCC gene expression with user-friendly interfaces [34]. The global differential gene expression landscape of HCC was built by analyzing the consistently differentially expressed genes across 15 public HCC gene expression datasets. In the study, we choose HCCDB18 to identify whether there existed significant difference of YTH domain protein expression between tumor samples and adjacent samples. We also displayed the co-expressed genes of YTH domain family in HCC samples, adjacent tissue samples, and normal liver samples, respectively.

\section{Kaplan-Meier plotter}

Kaplan-Meier plotter is a database assessing the relationship between gene expression with the survival trend of numerous cancer patients [35]. In our study, we evaluated the prognosis with HCC patients through means of OS and PFS curves. It showed statistical significance if the $\mathrm{p}$-value $<0.05$.

\section{cBioPortal}

cBioPortal provides a user-friendly analysis strategy concerning gene-disease associations on over 200 cancer cases [36]. In our study, we analyzed the coexpression profiles and genetic alterations of YTH domain family in HCC tissues by searching cBioPortal.

\section{STRING}

STRING was applied to explore potential proteinprotein interactions (PPIs). Furthermore, this database provides all resources concerning the interactive network of multiple proteins [37]. At the same time, we analyzed the YTH domain family member-associated PPI network using STRING and Cytoscape [38].

\section{GeneMANIA}

GeneMANIA has been applied in scientific research for many years and is very convenient for identifying protein-protein interactive networks [39]. Using GeneMANIA, we successfully identified the genes associated with the YTH domain family.

\section{WebGestalt}

WebGestalt aims to provide users with a better understanding of gene interpretation. Researchers can obtain enrichment results from this database [40]. In this study, we analyzed GO and KEGG enrichment pathways associated with the YTH domain family in HCC disease.

\section{TIMER}

TIMER is used to evaluate the connection between infiltrating immune cells and cancer cells, which could provide more rational strategies for an improved therapeutic response and prognosis [41]. We mainly performed correlation analysis between the expression 
of YTH domain family with immune cells using the TIMER database.

\section{Ualcan}

The Ualcan data portal can help to identify candidate biomarkers of specific cancer subtypes, with diagnostic, prognostic therapeutic implication [42]. In our study, we evaluated the relationship between the expression of YTH domain family and methylation levels.

\section{AUTHOR CONTRIBUTIONS}

Conception and design: Liu M and Zhao Z. Writing, review, and/or revision of the manuscript: $\mathrm{Xu} Z$, Cai $\mathrm{Y}$, Peng B and Yan Y. Administrative, technical, or material support: Liang Q and Yan Y. All authors approved final version of manuscript.

\section{CONFLICTS OF INTEREST}

This research was conducted in the absence of any commercial or financial relationships that could be construed as a potential conflicts of interest.

\section{FUNDING}

This study is supported by grants from the Natural Science Foundation of Hunan Province (2020JJ5953, 2020JJ5934, 2019JJ50932), the National Natural Science Foundation of China $(81803035,82104225)$, the China Postdoctoral Science Foundation (2021T140754, 2020M672521), and the Postdoctoral Science Foundation of Central South University (248485).

\section{REFERENCES}

1. Huang X, Qin F, Meng Q, Dong M. Protein tyrosine phosphatase receptor type D (PTPRD)-mediated signaling pathways for the potential treatment of hepatocellular carcinoma: a narrative review. Ann Transl Med. 2020; 8:1192.

https://doi.org/10.21037/atm-20-4733

PMID:33241041

2. Zhang J, Huang H, Bian J, Sang X, Xu Y, Lu X, Zhao H. Safety, feasibility, and efficacy of associating liver partition and portal vein ligation for staged hepatectomy in treating hepatocellular carcinoma: a systematic review. Ann Transl Med. 2020; 8:1246.

https://doi.org/10.21037/atm-20-2214 PMID:33178778

3. Singal AG, Lampertico $P$, Nahon P. Epidemiology and surveillance for hepatocellular carcinoma: New trends. J Hepatol. 2020; 72:250-61. https://doi.org/10.1016/i.jhep.2019.08.025 PMID:31954490

4. Feng $\mathrm{D}$, Wang $\mathrm{M}, \mathrm{Hu}$ J, Li S, Zhao S, Li H, Liu L. Prognostic value of the albumin-bilirubin grade in patients with hepatocellular carcinoma and other liver diseases. Ann Transl Med. 2020; 8:553.

https://doi.org/10.21037/atm.2020.02.116 PMID:32411776

5. Geng Y, Guan R, Hong W, Huang B, Liu P, Guo X, Hu S, Yu $M$, Hou B. Identification of m6A-related genes and m6A RNA methylation regulators in pancreatic cancer and their association with survival. Ann Transl Med. 2020; 8:387.

https://doi.org/10.21037/atm.2020.03.98 PMID:32355831

6. He $Y$, Xing J, Wang S, Xin S, Han Y, Zhang J. Increased m6A methylation level is associated with the progression of human abdominal aortic aneurysm. Ann Transl Med. 2019; 7:797. https://doi.org/10.21037/atm.2019.12.65 PMID:32042813

7. Huang $H$, Bai $Y$, Lu X, Xu Y, Zhao H, Sang X. N6methyladenosine associated prognostic model in hepatocellular carcinoma. Ann Transl Med. 2020; 8:633. https://doi.org/10.21037/atm-20-2894 PMID:32566570

8. Dai XY, Shi L, Li Z, Yang HY, Wei JF, Ding Q. Main N6Methyladenosine Readers: YTH Family Proteins in Cancers. Front Oncol. 2021; 11:635329.

https://doi.org/10.3389/fonc.2021.635329 PMID:33928028

9. Xu Z, Peng B, Cai Y, Wu G, Huang J, Gao M, Guo G, Zeng S, Gong Z, Yan Y. N6-methyladenosine RNA modification in cancer therapeutic resistance: Current status and perspectives. Biochem Pharmacol. 2020; 182:114258.

https://doi.org/10.1016/j.bcp.2020.114258

PMID:33017575

10. Zhang $X$, Wang F, Wang Z, Yang X, Yu H, Si S, Lu J, Zhou Z, Lu Q, Wang Z, Yang H. ALKBH5 promotes the proliferation of renal cell carcinoma by regulating AURKB expression in an $\mathrm{m}^{6} \mathrm{~A}$-dependent manner. Ann Transl Med. 2020; 8:646.

https://doi.org/10.21037/atm-20-3079

PMID:32566583

11. Zhang Z, Theler D, Kaminska KH, Hiller M, de la Grange $P$, Pudimat R, Rafalska I, Heinrich B, Bujnicki JM, Allain FH, Stamm S. The YTH domain is a novel RNA binding domain. J Biol Chem. 2010; 285:14701-10. https://doi.org/10.1074/jbc.M110.104711 PMID:20167602 
12. Liao $S$, Sun $H, X u$ C. YTH Domain: A Family of $\mathbf{N}^{6}$ methyladenosine $\left(\mathrm{m}^{6} \mathrm{~A}\right)$ Readers. Genomics Proteomics Bioinformatics. 2018; 16:99-107.

https://doi.org/10.1016/i.gpb.2018.04.002

PMID:29715522

13. Zhao BS, Roundtree IA, He C. Post-transcriptional gene regulation by mRNA modifications. Nat Rev Mol Cell Biol. 2017; 18:31-42.

https://doi.org/10.1038/nrm.2016.132

PMID:27808276

14. Nachtergaele $\mathrm{S}$, He $\mathrm{C}$. The emerging biology of RNA post-transcriptional modifications. RNA Biol. 2017; 14:156-63.

https://doi.org/10.1080/15476286.2016.1267096 PMID:27937535

15. Shi R, Ying $S$, Li Y, Zhu L, Wang $X$, Jin H. Linking the YTH domain to cancer: the importance of YTH family proteins in epigenetics. Cell Death Dis. 2021; 12:346. https://doi.org/10.1038/s41419-021-03625-8 PMID:33795663

16. Lin X, Chai G, Wu Y, Li J, Chen F, Liu J, Luo G, Tauler J, Du J, Lin S, He C, Wang $\mathrm{H}$. RNA $\mathrm{m}^{6} \mathrm{~A}$ methylation regulates the epithelial mesenchymal transition of cancer cells and translation of Snail. Nat Commun. 2019; 10:2065.

https://doi.org/10.1038/s41467-019-09865-9

PMID:31061416

17. Liu X, Qin J, Gao T, Li C, He B, Pan B, Xu X, Chen X, Zeng $\mathrm{K}, \mathrm{Xu} \mathrm{M}$, Zhu C, Pan Y, Sun H, et al. YTHDF1 Facilitates the Progression of Hepatocellular Carcinoma by Promoting FZD5 mRNA Translation in an m6ADependent Manner. Mol Ther Nucleic Acids. 2020; 22:750-65.

https://doi.org/10.1016/j.omtn.2020.09.036 PMID:33230473

18. Zhong L, Liao D, Zhang $M$, Zeng $C$, Li X, Zhang R, Ma H, Kang T. YTHDF2 suppresses cell proliferation and growth via destabilizing the EGFR mRNA in hepatocellular carcinoma. Cancer Lett. 2019; 442:252-61.

https://doi.org/10.1016/i.canlet.2018.11.006

PMID:30423408

19. Hou J, Zhang H, Liu J, Zhao Z, Wang J, Lu Z, Hu B, Zhou J, Zhao Z, Feng M, Zhang $H$, Shen B, Huang $X$, et al. YTHDF2 reduction fuels inflammation and vascular abnormalization in hepatocellular carcinoma. Mol Cancer. 2019; 18:163.

https://doi.org/10.1186/s12943-019-1082-3

PMID:31735169

20. Sun L, Song L, Wan Q, Wu G, Li X, Wang Y, Wang J, Liu Z, Zhong X, He X, Shen S, Pan X, Li A, et al. cMycmediated activation of serine biosynthesis pathway is critical for cancer progression under nutrient deprivation conditions. Cell Res. 2015; 25:429-44. https://doi.org/10.1038/cr.2015.33 PMID:25793315

21. Sangro B, Sarobe P, Hervás-Stubbs S, Melero I. Advances in immunotherapy for hepatocellular carcinoma. Nat Rev Gastroenterol Hepatol. 2021; 18:525-43.

https://doi.org/10.1038/s41575-021-00438-0 PMID:33850328

22. Yamaguchi $O$, Imai $H$, Minemura $H$, Suzuki $K$, Wasamoto S, Umeda Y, Osaki T, Kasahara N, Uchino J, Sugiyama T, Ishihara S, Ishii H, Naruse I, et al. Efficacy and safety of immune checkpoint inhibitor monotherapy in pretreated elderly patients with nonsmall cell lung cancer. Cancer Chemother Pharmacol. 2020; 85:761-71.

https://doi.org/10.1007/s00280-020-04055-7 PMID:32193618

23. Schett A, Rothschild SI, Curioni-Fontecedro A, Krähenbühl S, Früh M, Schmid S, Driessen C, Joerger M. Predictive impact of antibiotics in patients with advanced non small-cell lung cancer receiving immune checkpoint inhibitors : Antibiotics immune checkpoint inhibitors in advanced NSCLC. Cancer Chemother Pharmacol. 2020; 85:121-31.

https://doi.org/10.1007/s00280-019-03993-1 PMID:31745593

24. Bhoopalan SV, Cross SJ, Panetta JC, Triplett BM. Pharmacokinetics of alemtuzumab in pediatric patients undergoing ex vivo T-cell-depleted haploidentical hematopoietic cell transplantation. Cancer Chemother Pharmacol. 2020; 86:711-17.

https://doi.org/10.1007/s00280-020-04160-7 PMID:33037919

25. Ju $Q$, Li XM, Zhang $H$, Zhao YJ. BRCA1-Associated Protein Is a Potential Prognostic Biomarker and Is Correlated With Immune Infiltration in Liver Hepatocellular Carcinoma: A Pan-Cancer Analysis. Front Mol Biosci. 2020; 7:573619.

https://doi.org/10.3389/fmolb.2020.573619 PMID:33240929

26. Ju Q, Li X, Zhang $H$, Yan S, Li Y, Zhao Y. NFE2L2 Is a Potential Prognostic Biomarker and Is Correlated with Immune Infiltration in Brain Lower Grade Glioma: A Pan-Cancer Analysis. Oxid Med Cell Longev. 2020; 2020:3580719.

https://doi.org/10.1155/2020/3580719 PMID:33101586

27. Andrejeva G, Rathmell JC. Similarities and Distinctions of Cancer and Immune Metabolism in Inflammation and Tumors. Cell Metab. 2017; 26:49-70.

https://doi.org/10.1016/i.cmet.2017.06.004 PMID:28683294 
28. Yarchoan M, Johnson BA 3rd, Lutz ER, Laheru DA, Jaffee EM. Targeting neoantigens to augment antitumour immunity. Nat Rev Cancer. 2017; 17:209-22.

https://doi.org/10.1038/nrc.2016.154 PMID:28233802

29. Xu Y, He X, Deng J, Xiong L, Li Y, Zhang X, Chen W, Liu X, $\mathrm{Xu} X$. Comprehensive Analysis of the Immune Infiltrates and PD-L1 of $\mathrm{m}^{6} \mathrm{~A}$ RNA Methylation Regulators in Hepatocellular Carcinoma. Front Cell Dev Biol. 2021; 9:681745.

https://doi.org/10.3389/fcell.2021.681745

PMID:34277622

30. Shao XY, Dong J, Zhang H, Wu YS, Zheng L. Systematic Analyses of the Role of the Reader Protein of $\mathrm{N}^{6}$ Methyladenosine RNA Methylation, YTH Domain Family 2, in Liver Hepatocellular Carcinoma. Front Mol Biosci. 2020; 7:577460.

https://doi.org/10.3389/fmolb.2020.577460

PMID:33344502

31. Shi $Y$, Fan $S$, Wu M, Zuo Z, Li X, Jiang L, Shen $Q$, Xu P, Zeng L, Zhou Y, Huang Y, Yang Z, Zhou J, et al. YTHDF1 links hypoxia adaptation and non-small cell lung cancer progression. Nat Commun. 2019; 10:4892.

https://doi.org/10.1038/s41467-019-12801-6

PMID: $\underline{31653849}$

32. Díez-Villanueva A, Mallona I, Peinado MA. Wanderer, an interactive viewer to explore DNA methylation and gene expression data in human cancer. Epigenetics Chromatin. 2015; 8:22.

https://doi.org/10.1186/s13072-015-0014-8

PMID:26113876

33. Tang Z, Kang B, Li C, Chen T, Zhang Z. GEPIA2: an enhanced web server for large-scale expression profiling and interactive analysis. Nucleic Acids Res. 2019; 47:W556-60.

https://doi.org/10.1093/nar/gkz430

PMID: $\underline{31114875}$

34. Lian Q, Wang S, Zhang G, Wang D, Luo G, Tang J, Chen L, Gu J. HCCDB: A Database of Hepatocellular Carcinoma Expression Atlas. Genomics Proteomics Bioinformatics. 2018; 16:269-75. https://doi.org/10.1016/i.gpb.2018.07.003 PMID:30266410

35. Gyorffy B, Gyorffy A, Tulassay Z. [The problem of multiple testing and solutions for genome-wide studies]. Orv Hetil. 2005; 146:559-63.

PMID:15853065
36. Cerami E, Gao J, Dogrusoz U, Gross BE, Sumer SO, Aksoy BA, Jacobsen A, Byrne CJ, Heuer ML, Larsson E, Antipin $Y$, Reva B, Goldberg AP, et al. The cBio cancer genomics portal: an open platform for exploring multidimensional cancer genomics data. Cancer Discov. 2012; 2:401-04.

https://doi.org/10.1158/2159-8290.CD-12-0095

PMID:22588877

37. Szklarczyk D, Gable AL, Lyon D, Junge A, Wyder S, Huerta-Cepas J, Simonovic M, Doncheva NT, Morris JH, Bork $P$, Jensen $\amalg$, Mering CV. STRING v11: proteinprotein association networks with increased coverage, supporting functional discovery in genome-wide experimental datasets. Nucleic Acids Res. 2019; 47:D607-13.

https://doi.org/10.1093/nar/gky1131

PMID:30476243

38. Otasek D, Morris JH, Bouças J, Pico AR, Demchak B. Cytoscape Automation: empowering workflow-based network analysis. Genome Biol. 2019; 20:185.

https://doi.org/10.1186/s13059-019-1758-4 PMID: 31477170

39. Warde-Farley D, Donaldson SL, Comes O, Zuberi K, Badrawi R, Chao P, Franz M, Grouios C, Kazi F, Lopes CT, Maitland A, Mostafavi S, Montojo J, et al. The GeneMANIA prediction server: biological network integration for gene prioritization and predicting gene function. Nucleic Acids Res. 2010; 38:W214-20. https://doi.org/10.1093/nar/gkq537 PMID:20576703

40. Liao Y, Wang J, Jaehnig EJ, Shi Z, Zhang B. WebGestalt 2019: gene set analysis toolkit with revamped Uls and APIs. Nucleic Acids Res. 2019; 47:W199-205.

https://doi.org/10.1093/nar/gkz401

PMID:31114916

41. Li T, Fu J, Zeng Z, Cohen D, Li J, Chen Q, Li B, Liu XS. TIMER2.0 for analysis of tumor-infiltrating immune cells. Nucleic Acids Res. 2020; 48:W509-14.

https://doi.org/10.1093/nar/gkaa407

PMID: $\underline{32442275}$

42. Chandrashekar DS, Bashel B, Balasubramanya SA, Creighton CJ, Ponce-Rodriguez I, Chakravarthi BV, Varambally S. UALCAN: A Portal for Facilitating Tumor Subgroup Gene Expression and Survival Analyses. Neoplasia. 2017; 19:649-58.

https://doi.org/10.1016/j.neo.2017.05.002

PMID:28732212 


\section{SUPPLEMENTARY MATERIALS}

\section{Supplementary Figure}
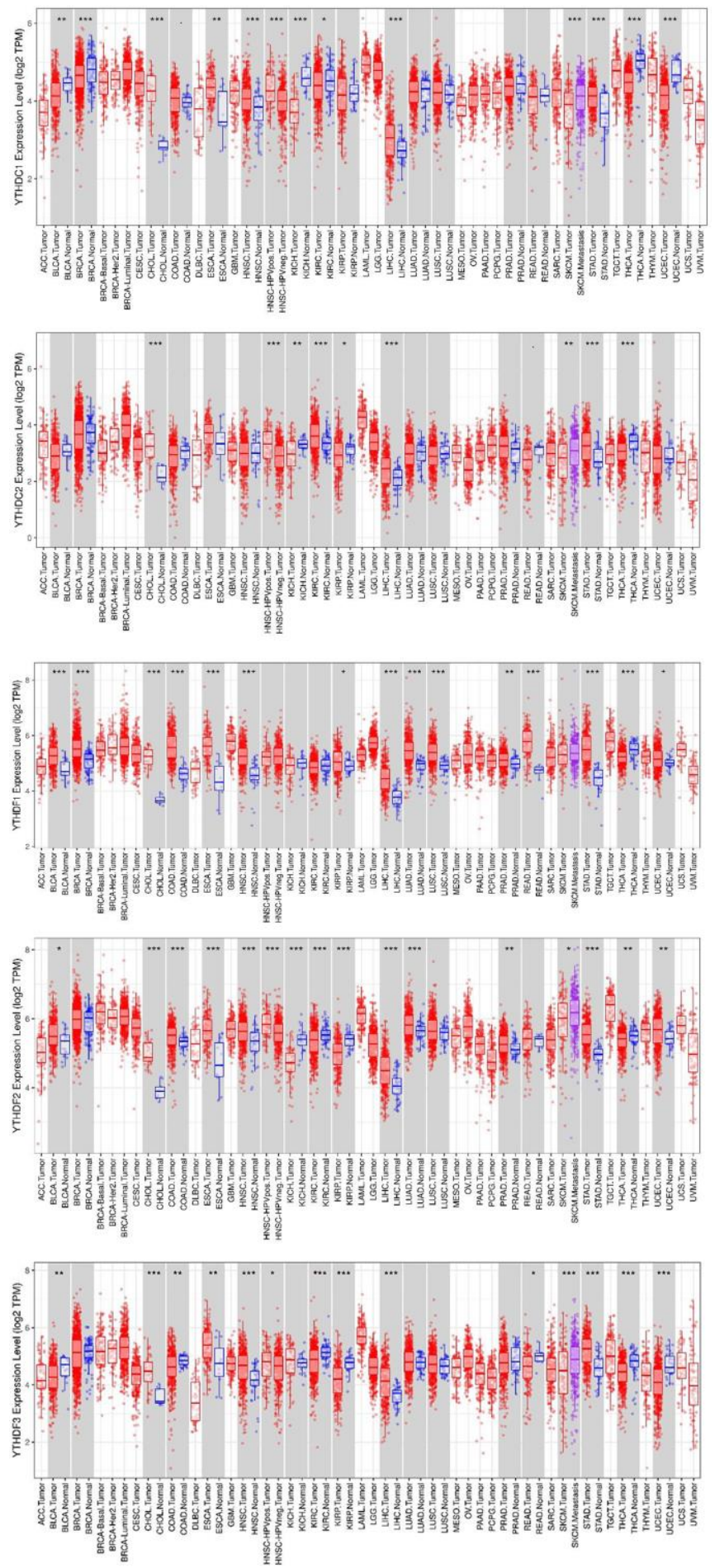

Supplementary Figure 1. YTH domain family expression in different cancer types via the tumor immune estimation resource (TIMER) database. 


\section{Supplementary Tables}

Please browse Full Text version to see the data of Supplementary Table 2.

Supplementary Table 1. The main databases applied to evaluate the expression and functions of YTH domain family in the biological process of ovarian serous carcinoma.

\begin{tabular}{lcccc}
\hline Databases & Authors & Publication date & Samples & URL \\
\hline Wanderer & Díez-Villanueva, A. et al. & 2015 & Tissues & $\underline{\text { http://maplab.imppc.org/wanderer/ }}$ \\
GEPIA2 & Tang Z. et al. & 2019 & Tissues & $\underline{\text { http://gepia.cancer-pku.cn/ }}$ \\
HCCDB & Lian Q. et al. & 2018 & Tissues & $\underline{\text { http://lifeome.net/database/hcdb }}$ \\
Kaplan-Meierplotter & Gyorffy B. et al. & 2005 & Tissues & $\underline{\text { http://kmplot.com/analysis/ }}$ \\
cBioPortal & Cerami E. et al. & 2012 & Tissues & $\underline{\text { http://www.cbioportal.org/ }}$ \\
STRING v11 & Szklarczyk D. et al. & 2019 & - & $\underline{\text { https://string-db.org/ }}$ \\
GeneMANIA & Warde-Farley D. et al. & 2010 & - & $\underline{\text { http://genemania.org/ }}$ \\
WebGestalt & Liao Y. et al. & 2019 & - & $\underline{\text { http://webgestalt.org/ }}$ \\
Timer 2.0 & Li T. et al. & 2020 & Tissues & $\underline{\text { https://cistrome.shinyapps.io/timer/ }}$ \\
DiseaseMeth 2.0 & Xiong Y. et al. & 2017 & Tissues & $\underline{\text { http://biobigdata.hrbmu.edu.cn/diseasemeth/ }}$ \\
\hline
\end{tabular}

GEPIA, Gene expression profiling interactive analysis; WebGestalt, the web-based GEne SeT AnaLysis Toolkit.

Supplementary Table 2. The database of cBioPortal applied to search the altered genes of the YTH domain family in tissues of hepatocellular carcinoma. 